\title{
Gaming on and off the Social Graph: The Social Structure of Facebook Games
}

\author{
Ben Kirman, Shaun Lawson and Conor Linehan \\ Lincoln Social Computing Research Centre \\ University of Lincoln \\ Lincoln, UK +44 1522837083 \\ \{bkirman|slawson|clinehan\}@lincoln.ac.uk
}

\begin{abstract}
Games built on Online Social Networks (OSNs) have become a phenomenon since 3rd party developer tools were exposed by OSNs such as Facebook. However, apart from their explosive popularity, little is known about the nature of the social networks that are built during play. In this paper, we present the findings of a network analysis study carried out on two Facebook applications, in comparison with a similar but stand-alone game. We found that games built both on and off a social graph exhibit similar social properties. Specifically, the distribution of playerto-player interactions decays as a power law with a similar exponent for the majority of players. For games built on the social network platform however, we find that the networks are characterised by a sharp cut-off, compared with the classically scale-free nature of the social network for the game not built on an existing social graph.
\end{abstract}

\section{INTRODUCTION}

It is hard to overstate the explosive growth in popularity that Online Social Networks (OSNs) have experienced in the last few years. The focus of the "Web 2.0" trend of social participation in the web, OSNs such as Facebook, Orkut, MySpace and Cyworld moved from being centred on content such as photographs or videos (e.g. Flickr and Youtube) to being centred on identity and the relationships between people. In the UK, connections to OSNs account for nearly $10 \%$ of all web requests and in both the UK and the USA, social networking sites are the most popular class of website except for search, and more popular even than pornography [1].

MySpace and Facebook are the two most popular OSNs [2] and both reach out to 3rd party developers by exposing interfaces (via OpenSocial[3] and the Facebook API[4]) that can be used to develop applications that integrate with the social graph maintained by each service. This has allowed thousands of developers to create applications that take advantage of a "ready-made" community. The platforms handle necessary services such as user management and verification, and in return the developers can provide highly integrated and socially useful applications that seamlessly integrate with the user experience on the site.

\section{A. Application Types}

Since its release in 2007, tens of thousands of applications have been deployed on the Facebook API. Popular themes for applications include gifting, self expression, recommendations, gestures, casual communication and friend comparisons [5].

Sharabi[6] classifies applications based on social purpose:
1) Self Presentation Tools: allow people to define their identities in terms of their favourite movies or their current mood.

2) Collective Identity Formation: that asks other people to define a person's identity - "Hot or Not" style games or applications that ask users to pick adjectives to describe their friends.

3) Phatic Communication Tools: are about maintaining social contact through small one way interactions, such as sending gifts, hugs and pokes.

\section{B. Games Applications}

Games are very popular on Facebook, and cross the whole range of classifications. It can be argued that in essence nearly all applications on Facebook are games, built around undirected play in a "Half-Real"[7] social sandbox.

More traditional game designs are also strongly represented in Facebook. For example the application Texas Holdem Poker has over 1.7 million daily users [8].

Although games built on platforms such as the Facebook API are clearly hugely popular, the question remains whether the style of play and types of players are fundamentally different when play is via a game on Facebook or outside of the formal strucutre of an OSN.

\section{OUR Social Games}

We compared three social games, both on and off the social graph of Facebook, and examined the nature of the social networks that were formed during play to look for differences both in structure and behaviour of the players.

\section{A. Hugged}

Hugged (http://apps.facebook.com/huggees [9]) is a simple example of an application used for Phatic communication between friends on Facebook. In this application, people simply choose an existing friend and a type of hug to send (e.g. Fuzzy Hug, Friendly Hug, Naughty Hug, etc.). The recipient receives this hug as a message along with an associated picture (often a bear, cat or other cute creature). Interactions are simply one time directed communication events that have no time constraints associated with them. 


\section{B. Fighters' Club}

Fighters' Club (http://apps.facebook.com/fightersc [9]) is a more complex social game application built on the Facebook platform. Players choose opponents from the whole set of Fighters' Club players and challenge them to a fight. There then follows a fixed time in which players may hit one another, and recruit the assistance of "supporters" to aid in their cause. After the time period is over, the winner is determined based on hits, supporters and the status of the players involved. The winner of a fight and their supporters gain points and prestige in the form of money and "Street Credit" which will make them more powerful in future conflicts. An important aspect to note is that players can pick fights with anyone within the social network of the game, not just those who are officially friends in Facebook. Players may also create and join formal groups with other Fighters' club players that compete in high scores based on the success of the individual members of the group.

\section{Familiars}

Familiars (http://www.familiars.eu [10]) is a social game based on a theme of collection. Players adopt virtual creatures (the eponymous "Familiars") and give them a series of tasks to be completed by other players. These tasks may be anything and are arbitrarily decided by players and described in terms of a short descriptive title. Other players then pick up familiars which have tasks they may feel they can contribute to. For example, a familiar with a task "What is your favourite holiday destination?" may be picked up by a player who can then add a contribution as a mix of text and an image, such as "Santorini, Greece". All interactions are associated with a location provided by self-report by the player on an interactive map, so in the example the contributing player may associate their message with the real location of Santorini.

1) Common Game Mechanics: In essence, both Fighters' Club and Familiars are very similar games in terms of mechanics. Both are centred around game events that require collaboration with other players. In the case of Fighters' Club, this is "Fights" which require "support" from other players. In Familiars the equivalent is "Tasks" that require "contributions". Although both games have additional layers of complexity (e.g. groups in Fighters' Club and location or images in Familiars), at their heart they are games about collaboration between groups of individuals in pursuit of mutual advantage.

In both Fighters' Club and Familiars, player score (Street Credit in FC) is a function of the social behaviour of the person within the game. The more a player contributes to existing events, the more points are awarded to the player. In Familiars the points are assigned based on social network indices within the game (for more information see [10], [11]), compared to Fighters' Club fixed points system where points are awarded based on the street credit of opposing players.

The main difference between the two games apart from theme is that Fighters' Club is explicitly built on top of a large existing social network (Facebook) where Familiars is completely stand-alone. Despite being built on an existing social network, Fighters' Club does not restrict interactions to be between players who have an existing connection in the social network (i.e. Friends). However as discussed in [9], it was common for new players to join the game after noticing a friend's activity within the game.

In neither game was the data for existing relationships between players available for analysis. In Fighters' Club existing relationship data was not recorded, and in Familiars it was unknown due to the lack of connection to existing social networks.

\section{Network Structure}

The structure of the social networks for all three games was analysed, using several standard network analysis methods, to identify the properties of the social graphs that were built during play. The game networks are made up of nodes (individual players within the sample) and edges (non-directional interactions between two players). For both Fighters' Club and Familiars, interactions are counted for every contribution to each game event. The sample for Familiars is based on 8 weeks of interaction data between all players from July-August 2008. The samples for both Hugged and Fighters' Club are based on one week's worth of interactions ending 1st April 2008 [9].

Table I shows some broad statistics about the structure of the networks that each game supports. The most striking difference is, of course, in scale. The applications deployed on the existing social network of Facebook attracted more players than the stand-alone application by several orders of magnitude. These are impressive numbers indeed, especially considering all but a couple of the most popular commercial online games attract less than $1 \mathrm{M}$ players [12].

\section{A. Clustering Coefficient}

The Clustering Coefficient shows the average number of connections each node has within its local graph neighbourhood. This measure identifies clustering based on how likely two neighbours of a player are to be connected to one another. For example, for every player $u$ that is adjacent to the set of nodes $V, \gamma$ is equal to the proportion of neighbours of each node $v$ that are also adjacent to $u$. The clustering coefficient is the average value of $\gamma$ for every node in the graph. A high $\gamma$ shows players are highly selective in their interactions, preferring to only interact with players that are known to one of their neighbours.

As the value of $\gamma$ increases, the graph is more likely to break up into several, small, tightly knit graphs (i.e. a Caveman World[13]). A value of $\gamma$ approaching 0 indicates interaction partners are selected randomly from the set of all possible players.

The average clustering coefficients for the networks analysed here shows a perhaps surprising trend for players in the larger games to be significantly more promiscuous in their choice of interaction partners within the game. The Familiars players were much more likely to choose to play with a select group of friends, and friends of friends, rather than playing 
TABLE I

Comparitive Network Properties

\begin{tabular}{l|c|c|c}
\hline Property & Familiars & Fighters' Club & Hugged \\
\hline Nodes $(N)$ & 157 & 143020 & 1322631 \\
Edges (Interactions) & 603 & 263112 & 1555597 \\
Size of Largest Subgraph & $147(93.6 \%)$ & $113614(79.4 \%)$ & $1169456(75.2 \%)$ \\
Avg Clustering Coefficient $(\gamma)$ & 0.471 & 0.042 & 0.053 \\
\hline
\end{tabular}

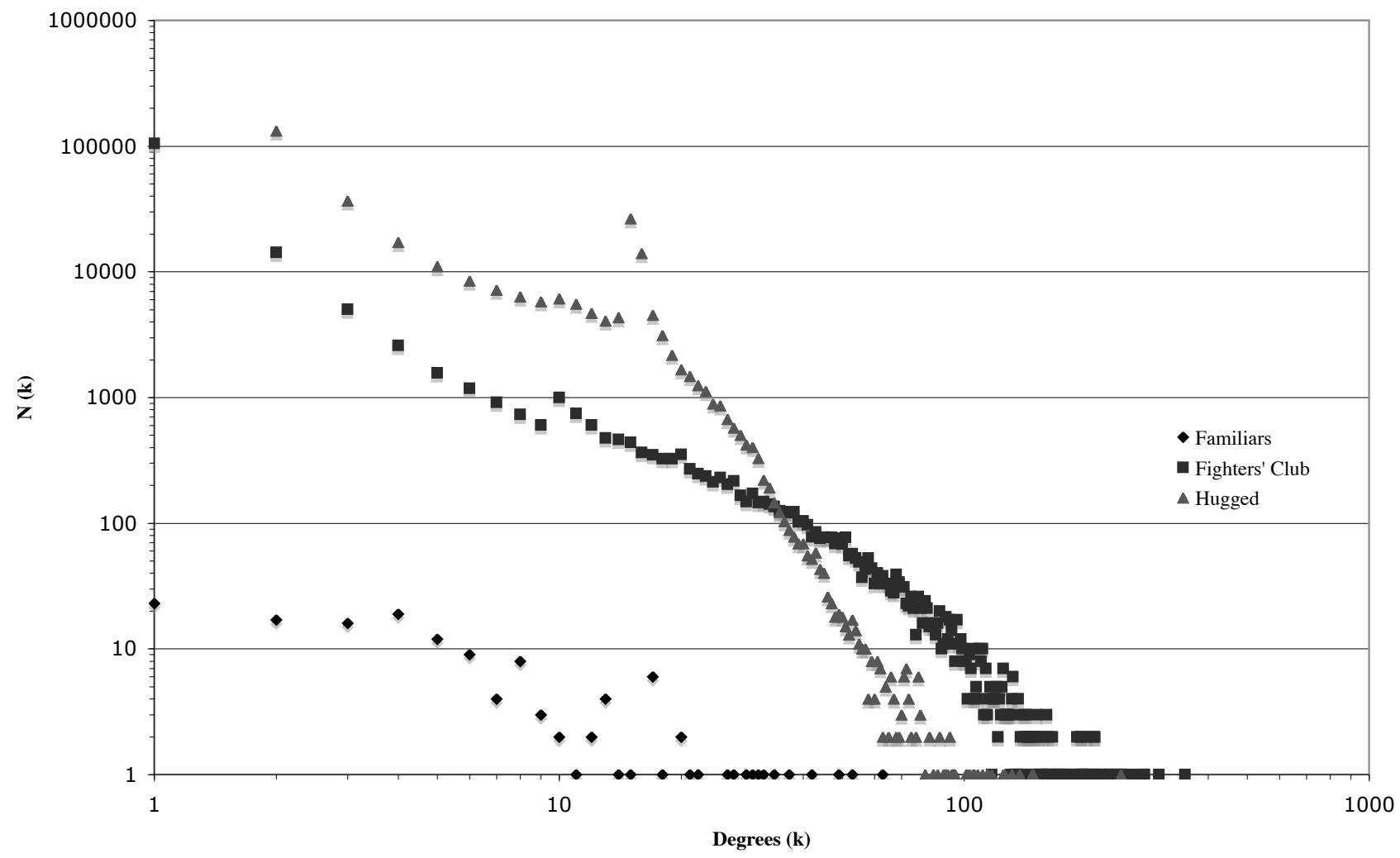

Fig. 1. Node Degrees Comparison

with just random partners. In both Fighters' Club and Hugged, during the one week sample players would seem to regularly play with a wide number of players regardless of relationship.

For Hugged specifically, the phatic, personal and emotive nature of the interactions (hugging) at first glance would not seem to be compatible with the scale of promiscuity. People are only limited to hugging players with which they have a formalised relationship (Facebook Friends), so the low incidence of clustering is explained by the lack of reciprocal relationships in the application. For example, a player may send hugs to many of their friends, however very few of those hug-ees go on to hug other people within the week studied.

\section{B. Small Worlds}

Small World networks are a peculiar kind of network graph where every node can trace a path to every other node in the network[13]. They are named for the famous "Small World" experiments carried out by Travers and Milgram in the late 1960's [14], which attempted to prove that everyone can trace a social connection to any random person on earth through a chain of mutual acquaintances. The most popular example of a small world network is the "Kevin Bacon Graph" that shows every movie actor is linked to every other movie actor through a chain of mutual film appearances [15], [16].

Familiars is the only game of the three that may be immediately classed as a small world network. Except for 10 players who never interacted with anyone, every node within the social graph can trace a path to every other (Average path length of just 2.31).

The Facebook games may well be classed as a small world, however the limited size of the sample is not adequate to make this proof (although as pointed out in [9], longer samples are so vast as to make analysis computationally unfeasible). Despite this, it is clear that both games are almost small worlds - the largest connected sub-graphs in both games include over 75\% of the player-base, and the next largest sub-graph for either game is many times smaller [9]. 


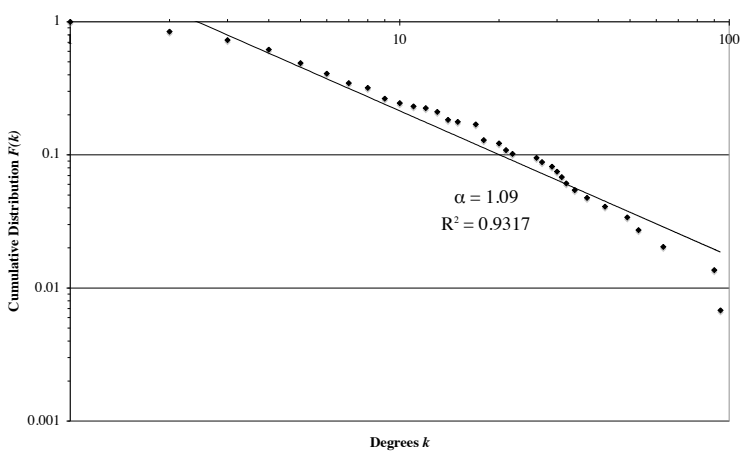

Fig. 2. Cumulative Distribution of $k$ in Familiars

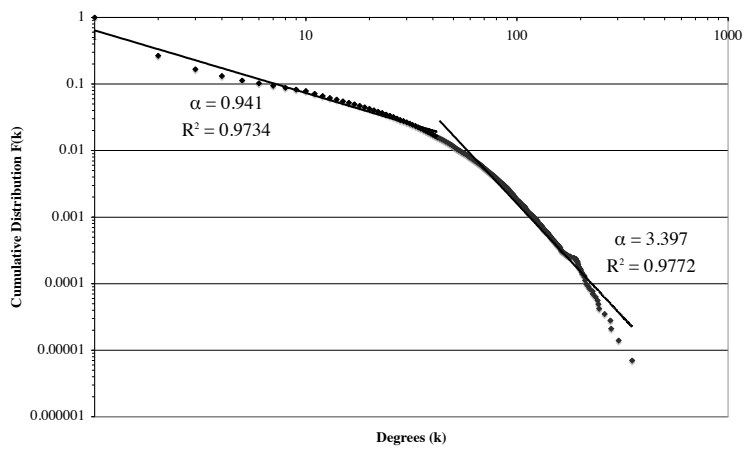

Fig. 3. Cumulative Distribution of $k$ in Fighters' Club

\section{Scaling IN Social Games}

Figure III shows a plot of the number of players each player had interacted with (Degrees $k$ ) in each game in a log-log scale. The value of $k$ for each node $u$ is defined as the number of distinct users that they have interacted with (either as sender or recipient of an event) at least once within the sample period. In other words, $k$ is the number of edges connected to node $u$. This values for $k$ are plotted against the frequency of $k$ $(N(k))$ for all nodes in the graph.

Here it can be observed that all three games appear to follow a power law (straight line) for scaling. In their original study, Nazir et al [9] did not address the question of scaling in Fighters' Club and Hugged, so we investigated this more deeply and in comparison with Familiars.

Scaling in networks are characterised by the way that many networks exhibit preferential connectivity as they grow [17]. In other words, as new nodes join an existing network, they are much more likely to connect to a highly connected node and therefore "the rich get richer". For Scale Free networks in particular this growth is characterised by a power-law decay of the cumulative distribution $P(k) \approx k^{-\alpha}$.

1) Scaling in Familiars: Figure IV-1 shows the cumulative distribution of $k$ in the social graph of Familiars. The trend line fits a power law with $\alpha=1.09$ and shows that the social network within Familiars can indeed by classified as a pure Scale Free network.

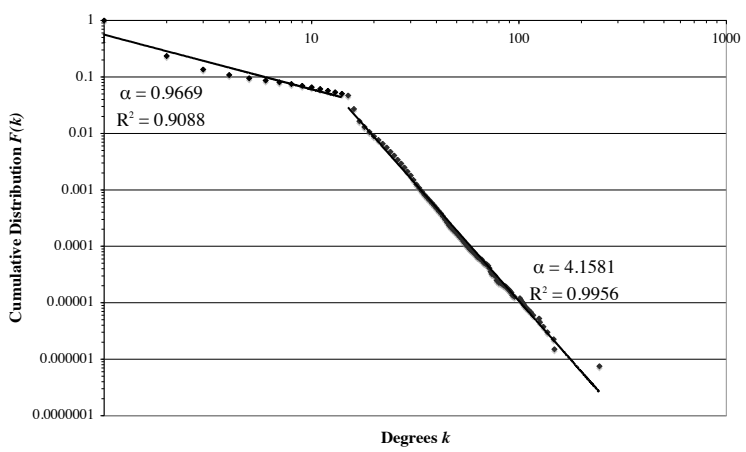

Fig. 4. Cumulative Distribution of $k$ in Hugged

2) Scaling in Fighters' Club: Figure IV-2 shows the cumulative distribution of Fighters' Club. As can be seen, for the range $k \leq 42$ the graph shows similar scaling properties ( $\alpha=0.941$ ) to the Familiars graph. Where $k>42$, the scaling shifts to $\alpha=3.397$.

3) Scaling in Hugged: Figure IV-3 shows the same distribution for Hugged. As with Fighters' club, the social graph for Hugged shows a strong sign of multi-scaling, with the range $k \leq 15$ showing $\alpha=0.9669$ and at $k>15$ having a very dramatic and sharp phase change with $\alpha=4.1581$.

\section{A. Facebook Games as Broad Scale Networks}

In [18], Amaral et al discuss several real world examples of social networks that exhibit the properties of broad scaling, including the graph of movie actor collaborations. In their analysis, broad scaling is a symptom of a hindrance in the process for preferential attachment [17]. The hindrance can be of two classes: Aging of vertices (e.g. an Actor retiring and therefore not receiving any more links); Increased Cost of attachment or Limited Capacity (e.g. An airport can't scale indefinitely to handle an infinite number of flights).

In the Facebook game examples it seems likely that there is a technical reason that caused the emergence of broad-scaling in those networks through limiting the capacity of the nodes.

In the Hugged application specifically, a player may only select a finite number of friends to hug at once, and the Facebook API limits the number of requests a player may make to friends in a 24 hour period $^{1}$. A single hug request to up to 15 players once a week would place that player in the first scale of users, whereas it appears that players are highly unlikely to repeat this after the time limit to requests had expired, explaining the particularly sharp change in the scaling exponent in Figure IV-3.

For Fighters' Club, the cause of the broad-scaling is less clear. In the application, players create fights that last, by default, 48 hours. The addition of supporters is done in two ways: first by manual invitation (limited in the same way as Hugged requests) and secondly by group memberships - fellow group members can always join fights and this is not limited

\footnotetext{
${ }^{1}$ At the moment this is 20 . See http://wiki.developers.facebook.com/index.php/Fb:multifriend-selector
} 
by the application, just the size of the groups. It is possible that the same technical limitation is the cause of the broadscaling in Hugged and Fighters' Club, except that in Fighters' Club the phase shift is less dramatic due to the group nature of the game, which allows additional supporters to join a fight without facing the same technical barrier.

\section{CONCLUSION}

Through network analysis we have analysed the structure of three social games, two that are built on the Facebook application platform, and one game that is mechanically similar but not built on top of the platform.

Building an application on top of an OSN such as Facebook can clearly give great rewards, particularly in reach. Compared to a similar application off the social graph, the Facebook games had significantly more users in a very short period. The tight integration with a social graph gives applications great possibilities for viral growth since the platform automatically exposes application usage to friends and acquaintances, offering an implicit recommendation for the application by the players.

Applications built both on and off the social graph such as Facebook exhibit very similar scaling properties for the majority of users, showing that the distribution of the most active players is comparable. This appears to hold true for all the games, but also phatic communication applications such as Hugged. This highlights what may be a standard pattern of behaviour within online social applications.

We have demonstrated that the reliance of an application on platforms such as Facebook API can have dramatic effects on the application's capability for social scaling due to limitations in the platform. Decisions taken by the OSN to limit the capability in this way are made for sensible reasons associated with the platform owner's concerns (i.e. preventing spam), but create sudden barriers that prevent the most hardcore and active users from using the applications to the level that they desire.

It is easy for application developers to be blinded by the scale of popularity their application receives. This study highlights the need for developers to be aware of how dramatic the effect of platform limitations can be for the most active segments of their player-base, and take steps to ameliorate these effects through application design.

\section{A. Future Work}

The sample size for Familiars was dwarfed by the enormous popularity of the games built on the Facebook platform. The scaling properties of the network do break through both the barriers experienced by the Facebook applications so we are confident of the significance of the comparison. However further study of more popular games not built on a social graph is desirable to be able to repeat these findings. Study of scaling properties for all social games (both on and off the social graph) is also necessary to support the finding that the power law decay for social games in general appears have exponent $\alpha \approx 1$.0. We are currently trialling three applications built on the Facebook platform, including a re-implementation of Familiars ${ }^{2}$ to allow for a more direct comparison, and an application for identity presentation ${ }^{3}$ that will allow us to study the social impact of all different classes of applications as defined by Sharabi [6] in addition to repeating the studies presented in this paper.

\section{ACKNOWLEDGEMENTS}

We would like to thank Atif Nazir, Saqib Raza and Chen-Nee Chuah for making the usage data of Fighters' Club and Hugged available for study [9] (See http://www.ece.ucdavis.edu/rubinet/data.html).

This work is part of the PASION Project, which is funded under the Presence II Initiative in the Future Emerging Technologies within the European Framework VI Programme

\section{REFERENCES}

[1] R. Goad, "Social networks overtake adult websites," Hitwise Intelligence Online, 2009.

[2] Hitwise, "Web trends," Accessed April 7th 2009. [Online]. Available: http://www.hitwise.com/datacenter

[3] Google, "Opensocial."

[4] Facebook, "Facebook api." [Online]. Available: http://developers.facebook.com

[5] M. Gjoka, M. Sirivianos, A. Markopoulou, and X. Yang, "Poking facebook: characterization of osn applications," in WOSP '08: Proceedings of the first workshop on Online social networks. New York, NY, USA: ACM, 2008, pp. 31-36.

[6] A. Sharabi, "Facebook applications trends report," November 19th 2007. [Online]. Available: http://no-mans-blog.com/2007/11/19/facebookapplications-trends-report-1/

[7] J. Juul, Half-Real: Video Games between Real Rules and Fictional Worlds. MIT Press, 2005.

[8] Adonomics, "Top applications." [Online]. Available: http://adonomics.com/leaderboard

[9] A. Nazir, S. Raza, and C.-N. Chuah, "Unveiling facebook: a measurement study of social network based applications," in IMC '08: Proceedings of the 8th ACM SIGCOMM conference on Internet measurement. New York, NY, USA: ACM, 2008, pp. 43-56.

[10] B. Kirman, S. Lawson, D. Rowland, F. Davide, F. Collovà, and S. Puglia, "Familiars - manipulating social networks with mobile gaming," in Proceedings of the Games Design and Technology Workshop and Conference (GDTW) 2008, Liverpool, 2008.

[11] F. Martino, A. Miotto, F. Davide, and L. Gamberini, "Exploring social network indices as cues to augment communication and to improve social practices." in 1st International Workshop on Maps Based Interaction in Social Networks (MapISNet '07), 2007.

[12] B. S. Woodcock, "Mmog chart." [Online]. Available: www.mmogchart.com

[13] D. J. Watts, Small Worlds - The Dynamics of Networks between Order and Randomness. Princeton University Press, 1999.

[14] J. Travers and S. Milgram, "An experimental study of the small world problem," Sociometry, vol. 32.4, pp. 425-443, 1969.

[15] D. J. Watts, Six Degrees: The Science of a Connected Age. Norton, 2003.

[16] P. Reynolds, “The oracle of bacon,” http://oracleofbacon.org/. [Online]. Available: http://oracleofbacon.org/

[17] A.-L. Barabasi and R. Albert, "Emergence of scaling in random networks," Science, vol. 286, p. 509, 1999. [Online]. Available: http://www.citebase.org/abstract?id=oai:arXiv.org:cond-mat/9910332

[18] L. A. N. Amaral, A. Scala, M. Barthlmy, and H. E. Stanley, Classes of small-world networks, 2000, vol. 97, no. 21. [Online]. Available: http://www.pnas.org/content/97/21/11149.abstract

\footnotetext{
${ }^{2}$ Magpies: http://apps.facebook.com/magpiemagpie

${ }^{3}$ Confusingly also called Familiars: http://apps.facebook.com/familiar
} 